\title{
Capturing Video Stream Audience Over IP Networks
}

Vitor Chaves de Oliveira

\author{
Paulo B. Lopes
}

Gunnar Bedicks Jr 


\title{
Capturing Video Stream Audience Over IP Networks
}

\author{
Vitor C. Oliveira, Paulo B. Lopes and Gunnar Bedicks Jr \\ Mackenzie Presbyterian University, São Paulo - SP, Brazil
}

\begin{abstract}
In recent years, the transmission of audiovisual content presented substantial growth in the context of ondemand delivery, made possible through networks based on Internet Protocol, IP. This increase, boosted by internet access omnipresence and new consumption habits of this content, makes this category of communication to be continuously rivaled with traditional broadcasting technologies of Television (TV) signals. Thus, developing techniques to assess the efficacy and risks that the use of IP networks for this purpose imposes becomes relevant. Moreover, while for the named Broadcast TV has technical mechanisms that ensure the quality of this communication are well established, the same does not occur for video stream over IP, or IP Stream. The reason for this is that, for one of them, its technology is equipped with quality guarantees based on a circuit-switching operation in a connection-oriented systematic. While the other, once the web is considered, is unable to ensure quality due to its packetswitching architecture. And this is what sets it as a model called 'best effort' which, besides the features to allow channel sharing and systems interconnection, enables precisely what makes its increase in its use for audiovisual transmission, the applicationoriented provision. Thereby attending the consumer's desire for on demand content, subsequently amplifies the role of what and who is on the edge, ultimately placing the user as protagonist. In this IP scenario, it is underlined the lack of mechanisms, related to the Internet, capable of recording two technical parameters that influence the commercial chain that sustains this entire ecosystem, they are: availability and audience. Keeping this in mind and with the goal of evaluating this new method for audiovisual offering, this work has developed methodologies to acquire such information at this consumption edge. And this has been accomplished through the construction of a software tool. It was implemented as an extension to one of the most used browsers, Google Chrome, to capture the audience of Internet videos on the leading supplier of videos exists today, Google's YouTube. In this work, the abstractions of considerations around the Quality of Service $(\mathrm{QoS})$ concept, is proposed to be interpreted as a measure of Quality of Experience (QoE) assimilated from time viewed compared against the total time of a video. This justification is founded on the concept that quality translates into a particular subjectivity for each user and their individual expectations, so a measure of time is shown to be an effective thermometer. This effectiveness is justified by a principle consolidated within TV broadcast for decades, audience measurement. Thus, the construction of something analogous to the 'People's meter' in a similar scheme of 'Television Rating Points', TRP, running to capture of information in order to convey assertiveness to all stakeholders involved in this new delivery method.
\end{abstract}

Keywords - IP Stream, Audience, Connection Availability, Quality of Service (QoS), Quality of Experience (QoE), Software Development.

\section{INTRODUCTION}

To assess the audience of a delivered content over TV Broadcast it is used an equipment called People meter or Audience meter. This is installed at the home of certain viewers and connected to the TV continuously recording and sending information to a central regarding which channel is being watched, at what time and for how long. These members are chosen to have such devices installed in their homes so that their measures represent a sample of the whole society or people segment to be monitored. This complete equipment scheme is called Television Rating Points (TRP), or Gauging points for Television Audience, which aggregates all this measuring system. Thus, it estimates statistically the number of successful audiovisual content watched. The importance of measuring the audience is justified because it is the engine of the commercial part of a broadcaster station to choose which programs to invest or not and what impact or the reachability of advertising campaigns. Existing thus a significant commercial interest for pricing purposes of transmission periods in this and other segments, such as IP Streaming. [1][2]

\section{REQUIREMENTS ANALYSIS}

To capture Video Stream Audience, it was designed a solution considering aforementioned importance of obtaining this information at the user end of the video delivered via IP from the largest online media provider in the Internet, i.e. YouTube [3][4]. Also, it was delineated that the application is compatible with one of the most widely used browsers, Google Chrome [5]. Considering the requirements, in order to capture the audience of an audiovisual content it was decided on a project in which the program should be composed of two parts, described as follows. The client part or Front-end, it is an application to be installed in the browser in the format of an extension that could be installed on any device with the Google Chrome browser. This collects and sends certain data to a centralizer. These data are statistics (measures and information) of videos accessed, or viewed by users. It is emphasized that this is done from data present in the public codes of the website when a YouTube video page is accessed, time which the application is active. Such selected data which is captured and sent automatically to the centralizer are: Watched Time; Total Video Time; Video URL; Number of Video Views and Number of Channel Subscribers. In addition, other data considered relevant is captured through a brief questionnaire for completion by the user when the software is installed. These are: City; Age and Gender. The part of the centralizer, or server that receives, stores, manipulates and displays all these data, is called the back-end. Software that acts as a server on the Internet, which provides a service that receives, saves, records and consults the data captured by segmenting them in database tables.

\section{SYSTEM FUNCTIONING}

To develop a software that is able to interact with the socalled API, Application Programming Interface, or Youtube Application Programming Interface JavaScript was selected. And to store the data used a MySQL database, and hence the Structured Query Language to manipulate and process the 
data. The following Figure 1 displays the main parts of the code that was implemented for the Front-end which is the extension installed on the user device browser to monitor audiovisual content. Meanwhile Figure 2 shows the Software Operation

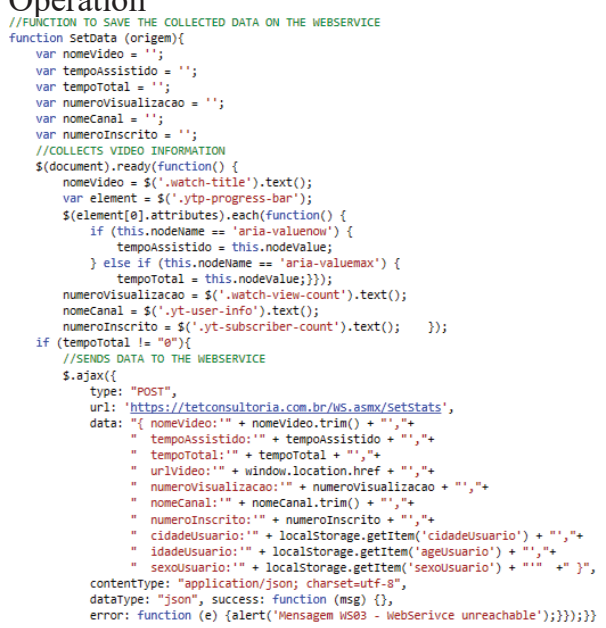

Flowchart.

Fig. 1. Extension core coding demonstrating the capture of monitored data and dispatching it to the Back-end Web service.

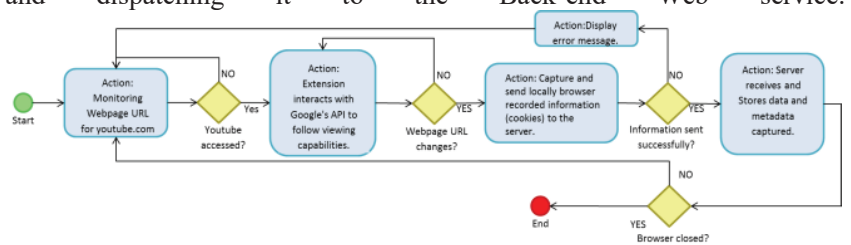

Fig. 2. Software Operation Flowchart

For the developed application, first the user needs via Chrome browser, access the Internet at 'Chrome Web Store Extensions' or 'Chrome Extension Store'. Once there, search for 'Youtube Data Stats' which is name designated to this software, the icon and application described in Figure 3 will appear to be installed on the user's browser, available by accessing the address: 'https://chrome.google.com/webstore/detail/youtube-datastats/nmnaacknlpckjpccicgekcnogaeoaipi'.

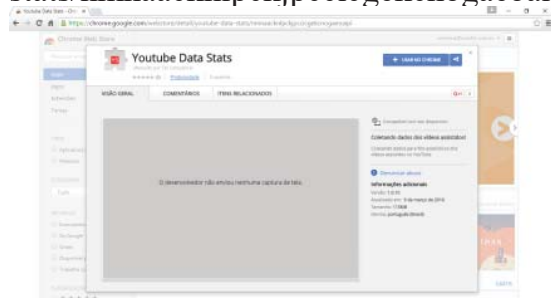

Fig. 3. Webpage to install the extension from the 'Chrome Web Store Extensions'.

\section{RESULTS AND CONCLUSION}

In order to detail the results from the monitoring carried out, then it is shown, in Figure 4, clearly all data captured from two tests sent to the back-end. From left to right we have the columns with the name information, Assisted Time, Total Time, URL, View, Canal, Subscribers, City, Age, Gender, Date.

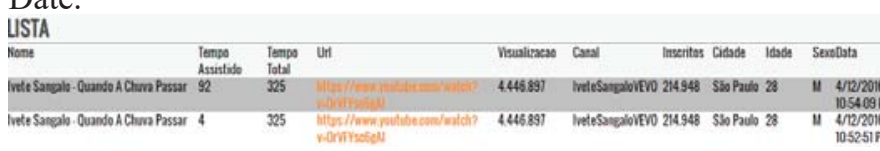

Fig. 4. Detailing search results from the database with the captured data at the Software's Back-end.

The technical and commercial motivation for verifying the audience is the same as the traditional TV and that the IP demand for content grows daily. The results show that this is a tool that meets this feature, allowing to bring to the IP world some of the assertiveness present only on TV so far, according to the literature. [6] [7] [8] [9]

\section{ACKNOWLEDGMENT}

The researchers thank the scholarship support offered by Mackenzie Presbyterian University.

\section{REFERENCES}

[1] IBOPE, 2016. Kantar IBOPE Media. Audiência de TV. Available at: $<$ https://www.kantaribopemedia.com/solucoes-type/audiencia-detv/>. Accessed on: June, 10, 2016.

[2] GFK, 2016. GFK Brasil. Overview. Available at: $<$ http://www.gfk.com/pt-br/sobre-a-gfk/overview/>. Accessed on: June, 10, 2016.

[3] Statista, 2015 A. Statista Inc. Statistics and facts about YouTube. Available at: <http://www.statista.com/topics/2019/youtube/>. Accessed on: June, 10, 2016.

[4] Statista, 2015 B. Statista Inc. Global market share held by the leading internet browsers from January 2012 to December 2015. Available at: $<$ http://www.statista.com/statistics/268254/market-share-of-internetbrowsers-worldwide-since-2009/>. Accessed on: June, 10, 2016.

[5] Statista, 2016. Statista Inc. YouTube - Statista Dossier. Available at: $<$ http://www.statista.com/topics/2019/youtube/>. Accessed on: June, 10, 2016.

[6] Oliveira, V. C., Bedicks Jr., G. and Akamine, C.; 2015. METHODOLOGY TO ASSES IP CONNECTION AVAILABILITY: A PREREQUISITE FOR FEASIBLE VIDEO STREAM THROUGH CDNs. Journal of Computer Science. ISSN: 1549-3636. Vol. 11, Issue 2, p. 426-437. DOI: 10.3844/jcssp.2015.426.437.

[7] Oliveira, V. C., Bedicks Jr., G. and Akamine, C.; 2015 B. IP VIDEO DELIVERY COST: AN ANALYSIS ON CHANNEL AVAILABILITY FOR LIVE AND NON-LIVE STREAM EVENTS In: 2015 - 64th - IEEE Broadcast Symposium, 2015, Orlando, FL, USA. 2015 - 64th - IEEE Broadcast Symposium Technical Program. New York, NY, USA: IEEE Institute of Electrical and Electronics Engineers / IEEE Broadcast Technology Society, IEEE BTS, 2015. v. 64.

[8] Doeven, J.; 2013. TRENDS IN BROADCASTING: AN OVERVIEW OF DEVELOPMENTS. ITU - International Telecommunication Union. Telecommunication Development Bureau. Available at $<$ http://www.itu.int/en/ITU-D/Technology/Documents/Broadcasting /TrendsinBroadcasting.pdf $>$. Accessed on: June, 10, 2016.

[9] Babatunde, O. and Al-Debagy, O.; 2014. A Comparative Review Of Internet Protocol Version 4 (IPv4) and Internet Protocol Version 6 (IPv6). International Journal of Computer Trends and Technology (IJCTT). Vol. 13, Number 1, p. 10-13, Jul 2014. ISSN: 2231-5381. Available at: http://arxiv.org/ftp/arxiv/papers/1407/1407.2717.pdf

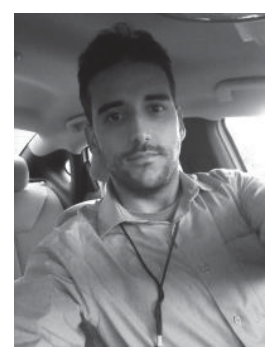

Vitor Chaves de Oliveira Engineering - Coach IT Group Inc. / UNISAL / DeVry Metrocamp / SET Vitor is a Brazilian Professor, Consultant and Researcher. PhD student in Electrical Engineering (Mackenzie), Master (MSc) in Electrical Engineering (PUCC), Specialist (MBA) in Electrotechnical Engineering and Power Systems (UNISAL), has a Bachelor's (BSc) Degree in Computer Science (UNISAL) and is an Informatics Technician (SENAI). College Professor working in the areas of Electrical Engineering and Computing at the Salesian University Center of São Paulo (UNISAL) and at the Metropolitan Integrated College of Campinas (DeVry Metrocamp); Magazines Editor (in Broadcast Engineering ) / Chair \& Manager for the SET EXPO Technology Conference for the Brazilian Society of 
Television Engineering (SET); Consultant in IT/ERP/Telecom for the 'Coach IT Group Inc.'. Is a book author in Cloud Computing area and has published dozens of articles in Scientific Journals and at National and International Conferences. His research interests are focused on Telecommunications: IP Networks, Radio Broadcasting (Digital TV), Electromagnetic Theory, Control Algorithms, Mathematical Systems Modeling, Virtualization and Energy Efficiency.

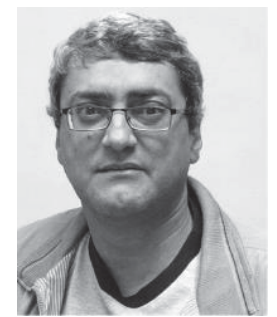

Paulo Batista Lopes Coordinator of the Graduate Program in Electrical Engineering and Computing Mackenzie Presbyterian University. A graduate of the Federal University of Rio de Janeiro (1978), with a Master's in Electrical Engineering from the same university (1981) and a doctorate in Electrical Engineering from Concordia University (1985). $\mathrm{He}$ is currently the Coordinator of the Graduate Program in Electrical Engineering and Computing at the Mackenzie Presbyterian University and a Reviewer of periodicals on Circuits, Systems and Signal Processing. He has experience in the field of Electrical Engineering, with the emphasis on Telecommunications, having worked in the industry for over 20 years. He is involved primarily with the following topics: Signals Processing, Active Filters, Modulation Systems, etc.

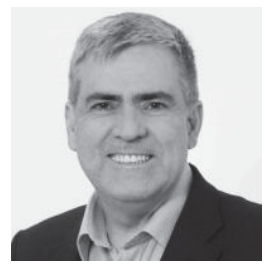

Gunnar Beddicks: CTO at EAD Associação Administradora do Processo de Redistribuição e Digitalização de Canais de TV e RTV/ Chief Scientist at the Digital TV Research Centre of the Universidade Presbiteriana Mackenzie in Sao Paulo Gunnar Bedicks is one of Brazil's most distinguished experts in developing, deploying and evangelizing for digital TV. In the last decade, he has visited 32 countries around the world to discuss their switch from analog to digital television. In 2013, President of Brazil Dilma Russef awarded Dr. Bedicks the highest honor in Brazilian diplomacy, the Medal of the Order of Rio Branco, for his achievements. Besides being Chief Scientist at the Digital TV Research Centre of the Universidade Presbiteriana Mackenzie in Sao Paulo, he teaches postgraduate, graduate and undergraduate courses in Digital Communications at the same university. He was one of the lead engineers for the testing and research that led the government of Brazil to adopt digital TV in 2004. His publications include more than a dozen scientific articles, and he has presented at technical conferences in Brazil, USA, Canada, Korea, Japan, China, Germany, France, Sweeden, Switzerland, Italy, Spain, South Africa, Botswana, Angola, Mozambique, Lesotho, Mauritus, Argentina, Chile, Colombia, Cuba, Costa Rica, Paraguay, Peru, Venezuela and Bolivia.

Dr. Bedicks has more than 20 years of experience in assisting technology corporations in Brazil, Germany, France, Italy and the USA with the research and development of components and products for the digital TV market. He is also an expert in broadcasting and telecommunication policies, dealing with certification agencies, standardization organizations, patents offices and government regulators. He is skilled at organizing projects, managing a team of experts and writing up project results. His degrees include a Ph.D. in Electrical Engineering and an M.B.A. in Administration from the Universidade de São Paulo, as well as a B.E. in Telecommunications Engineering from the Instituto Nacional de Telecomunicações in Santa Rita do Sapucai in Brazil. He holds seven patents and is fluent in Portuguese, English and German.

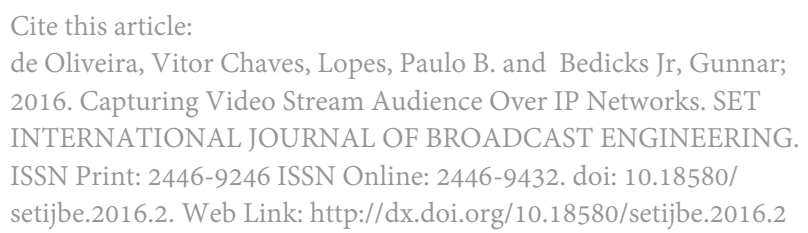

